\title{
Generalized Elitzur's Theorem and Dimensional Reductions
}

\author{
C. D. Batista and Zohar Nussinov \\ Theoretical Division, Los Alamos National Laboratory, Los Alamos, NM 87545
}

(Dated: Received November 4, 2018)

\begin{abstract}
We extend Elitzur's theorem to systems with symmetries intermediate between global and local. In general, our theorem formalizes the idea of dimensional reduction. We apply the results of this generalization to many systems that are of current interest. These include liquid crystalline phases of Quantum Hall systems, orbital systems, geometrically frustrated spin lattices, Bose metals, and models of superconducting arrays.

PACS numbers: 71.10.-w, 71.45.-d, 77.28.+d
\end{abstract}

\section{INTRODUCTION}

Symmetry occupies a central and indispensable role in physics. Amongst others, it is the key concept behind the theory of phase transitions and critical phenomena. Most of the known phase transitions break one or more symmetries. When symmetry breaking occurs, the mean value of a quantity which is not invariant under the symmetry group of the theory becomes "spontaneously" non-zero in the broken symmetry phase. Such a quantity is called the order parameter. As well appreciated, the dimension $D$ of the system and the symmetries of the order parameter characterize different universality classes and play a pivotal role in determining whether a particular phase transition may or may not exist. In particular, zero or one dimensional theories with short range interactions cannot exhibit a phase transition at any finite temperature. Additionally, the Mermin-Wagner theorem [1] states that a continuous symmetry cannot be spontaneously broken at any finite temperature for two-dimensional theories with finite range interactions. On the other hand, Elitzur [2] demonstrated that a spontaneous breaking of a local symmetry is not possible. Below, we will show that Elitzur's theorem is a consequence of a reduction to zero of the effective dimension of the gauge invariant theory. Moreover, we will show that from the point of view of the non-invariant gauge fields, the presence of a " $d$ dimensional gauge symmetry" (see definition below) reduces the effective dimension of the theory from $D$ to $d$. The dimension $d$ is intermediate between local gauge systems $(d=0)$ and global gauge symmetries $(d=D)$.

How is it possible that symmetry not only determines the nature of the transition but also its effective dimension? The answer to this question requires a definition of what we mean by local and " $d$-dimensional gauge symmetries". A local gauge symmetry has its roots in a group of operations which leave the theory invariant and in which the minimum nonempty set of fields influenced by any of these operations occupies a finite spatial region. A " $d$-dimensional gauge symmetry" is any symmetry operation which leaves the Hamiltonian (or action) invariant such that the minimum non-empty set of fields which are changed under the symmetry operation occupies a $d$-dimensional region. In accord with this definition, a local gauge symmetry corresponds to the particular case $d=0$. The above question is motivated by the recent interest in a considerable number of theories that are invariant under a $d$-dimensional gauge group with dimension $d \geq 1$
$[3,4,5,6,7,8,9,10]$. As was already noticed [3, 4, 5], some aspects of these theories indicate that the dimension is effectively reduced. In this paper we formalize this notion by proving a generalized Elitzur's theorem for any value of $d$.

We will prove that the mean value of a local quantity which is non-invariant under a $d$-dimensional gauge group can be bounded by another mean value of the same quantity which is computed for an effective $d$-dimensional theory. The gauge transformations become a global symmetry of the effective theory and the range of the interactions in the original theory is preserved. This proof formalizes the idea of "dimensional reduction" and gives rise to four important corollaries. The first corollary is Elitzur's theorem. The second states that a $d=1$ gauge symmetry cannot be broken at any finite temperature if the the theory only includes finite range interactions. The third corollary is a natural consequence of the MerminWagner theorem: a continuous $d=2$ gauge symmetry cannot be broken at any finite temperature if the interactions are of finite range. The fourth corollary extends Elitzur's theorem to certain systems in which at least an emergent low energy gauge-like symmetry appears. By emergent symmetry we mean a group transformations which become a symmetry of the Hamiltonian only within its lowest-energy subspace [11].

Recently, theories containing $d$-dimensional gauge symmetries with $d \geq 1$ became the focus of attention in different areas of physics. The Kugel-Khomskii model that describes the interplay between spin and orbital degrees of freedom in transition metals as well as orbital only variants embodying the Jahn-Teller interactions often display $d=1,2$ gauge-like symmetries [6, 7, 8, 9, 10]. The well known planar orbital compass model [6, 7, 10] is invariant under a $d=1 Z_{2}$-gauge group. The two-dimensional frustrated Ising magnet studied by $\mathrm{Xu}$ and Moore [4, 5] provides another example of a $d=1$ $Z_{2}$-gauge invariant theory. There are also examples of frustrated magnets [12] for which the $d=1 Z_{2}$-gauge group is an emergent symmetry [11]. Here, the dimensional reduction may occur at low energies. Similar symmetries appear in ring exchange Bose metals [13], Quantum Hall liquid crystalline phases [14], [15], lipid bilayers with intercalated DNA strands [16], and "sliding Luttinger liquids" [17].

The physical roots of our theorem and its corollaries are precisely the same fluctuations which generically inhibit order in low dimensions. Just as domain wall entropy eradicates any viable order in one dimensional systems having short range interactions, domain walls on different sliding phases of many 
of the above examples inhibit discrete $d=1$ gauge symmetry breaking at any finite temperature.Similar considerations apply to continuous $\mathrm{d}=1,2$ dimensional gauge symmetry systems whose order is destroyed by low energy excitations.

\section{A GENERALIZATION OF ELITZUR'S THEOREM}

We will start by considering the case of classical theories. As we will see later, the generalization to the quantum case is very simple. Before enunciating our theorem it is convenient to give a more precise formal definition of a $d$-dimensional gauge group. From now on we will consider theories which are defined on a lattice $\Lambda$. The extension to the continuum case is straightforward. A $d$-dimensional gauge symmetry of a theory characterized by a Hamiltonian $H$ is a group of symmetry transformations of $H$ such that the minimal non-empty set of fields $\phi_{\mathbf{i}}$ that is changed under the group operations occupies a $d$-dimensional subset $\mathcal{C} \subset \Lambda$. The index i denotes the sites of the lattice $\Lambda$. For instance, if a spin theory is invariant under flipping each individual spin the corresponding gauge symmetry is zero-dimensional or local. Of course flipping a chain of spins is also a symmetry, but the chain is not the minimal non-trivial subset of spins that can be flipped. In general, these transformations can be expressed as:

$$
\mathbf{U}_{l k}=\prod_{\mathbf{i} \in \mathcal{C}_{l}} \mathbf{g}_{\mathbf{i} k}
$$

where $\mathcal{C}_{l}$ denotes the subregion $l, \mathcal{C}_{l} \subset \Lambda$, and $\Lambda=\bigcup_{l} \mathcal{C}_{l}$.

Theorem: The absolute mean value of any local quantity (i.e. involving only a finite number of fields) which is not invariant under a d-dimensional gauge symmetry group $G$ of the D-dimensional Hamiltonian $H$ is equal or smaller than the absolute mean value of the same quantity computed for a $d$-dimensional Hamiltonian $\bar{H}$ which is globally invariant under the group $G$ and preserves the range of the interactions in $H$. Non invariant here means that the quantity under consideration, $f\left(\phi_{\mathbf{i}}\right)$, has no invariant component:

$$
\sum_{k} f\left[\mathbf{g}_{\mathbf{i} k}\left(\phi_{\mathbf{i}}\right)\right]=0
$$

For a continuous group Eq. (2) has to be replaced by $\int f\left[\mathbf{g}_{\mathbf{i}}\left(\phi_{\mathbf{i}}\right)\right] d \mathbf{g}=0$. To determine if there is a spontaneous symmetry breaking in the thermodynamic limit, the mean value of $f\left(\phi_{i}\right)$ has to be computed in the following way:

$$
\left\langle f\left(\phi_{\mathbf{i}}\right)\right\rangle=\lim _{h \rightarrow 0} \lim _{N \rightarrow \infty}\left\langle f\left(\phi_{\mathbf{i}}\right)\right\rangle_{h, N},
$$

where $\left\langle f\left(\phi_{i}\right)\right\rangle_{h, N}$ is the mean value of $f\left(\phi_{i}\right)$ computed on finite lattice of $N$ sites and in the presence of a symmetry breaking field $h$. Since $\Lambda=\bigcup_{l} \mathcal{C}_{l}$, the site $\mathbf{i}$ belongs at least to one set $\mathcal{C}_{j}$. It is convenient to rename the fields in the following way: $\phi_{\mathbf{i}}=\psi_{\mathbf{i}}$ if $\mathbf{i} \notin \mathcal{C}_{j}$ and $\phi_{\mathbf{i}}=\eta_{\mathbf{i}}$ if $\mathbf{i} \in \mathcal{C}_{j}$. The mean value $\left\langle f\left(\phi_{\mathbf{i}}\right)\right\rangle_{h, V}$ is given by:

$$
\begin{aligned}
& \left\langle f\left(\phi_{\mathbf{i}}\right)\right\rangle_{h, N}=\frac{\sum_{\left\{\phi_{\mathbf{i}}\right\}} f\left(\phi_{\mathbf{i}}\right) e^{-\beta H(\phi)} e^{-\beta h \sum_{\mathbf{i}} \phi_{\mathbf{i}}}}{\sum_{\left\{\phi_{\mathbf{i}}\right\}} e^{-\beta H(\phi)-\beta h \sum_{\mathbf{i}} \phi_{\mathbf{i}}}}= \\
& \frac{\sum_{\left\{\psi_{\mathbf{i}}\right\}} z_{\{\psi\}} e^{-\beta h \sum_{\mathbf{i} \notin \mathcal{C}_{j}} \psi_{\mathbf{i}}\left[\frac{\sum_{\left\{\eta_{\mathbf{i}}\right\}} f\left(\eta_{\mathbf{i}}\right) e^{-\beta H(\phi)-\beta h \sum_{\mathbf{i} \in \mathcal{C}_{j}} \eta_{\mathbf{i}}}}{z_{\{\psi\}}}\right]}}{\sum_{\left\{\psi_{\mathbf{i}}\right\}} z_{\{\psi\}} e^{-\beta h \sum_{\mathbf{i} \notin \mathcal{C}_{j}} \psi_{\mathbf{i}}}}
\end{aligned}
$$

with,

$$
z_{\{\psi\}}=\sum_{\left\{\eta_{\mathbf{i}}\right\}} e^{-\beta H(\psi, \eta)-\beta h \sum_{\mathbf{i} \in \mathcal{C}_{j}} \eta_{\mathbf{i}}} .
$$

From Eq. (4):

$$
\left|\left\langle f\left(\phi_{\mathbf{i}}\right)\right\rangle_{h, N}\right| \leq\left|\frac{\sum_{\left\{\eta_{\mathbf{i}}\right\}} f\left(\eta_{\mathbf{i}}\right) e^{-\beta H(\bar{\psi}, \eta)-\beta h \sum_{\mathbf{i} \in \mathcal{C}_{j}} \eta_{\mathbf{i}}}}{z_{\{\bar{\psi}\}}}\right|,
$$

where $\{\bar{\psi}\}$ is the particular configuration of fields $\psi_{\mathbf{i}}$ that maximizes the expression between brackets in Eq. (4). $H(\bar{\psi}, \eta)$ is a $d$-dimensional Hamiltonian for the field variables $\eta$ which is invariant under the global symmetry group $G_{j}$ of transformations $\mathbf{U}_{j k}$ over the field $\eta$. We can define $\bar{H}(\eta) \equiv H(\bar{\psi}, \eta)$. The range of the interactions between the $\eta$-fields in $\bar{H}(\eta)$ is clearly the same as the range of the interactions between the $\phi$-fields in $H(\phi)$. This completes the demonstration of our theorem. Note that the "frozen" variables $\bar{\psi}_{\mathbf{i}}$ act like external fields in $\bar{H}(\eta)$ which do not break the global symmetry group of transformations $\mathbf{U}_{j k}$.

Corollary I: Elitzur's theorem. Any local quantity (i.e. involving only a finite number of fields) which is not gauge invariant under a local or zero-dimensional gauge group has a vanishing mean value at any finite temperature. This is a direct consequence of Eq. (4) and the fact that $\bar{H}(\eta)$ is a zerodimensional Hamiltonian.

Corollary II. Any local quantity which is not gauge invariant under a one-dimensional gauge group has a vanishing mean value at any finite temperature for systems with finite range interactions. This is a consequence of Eq. 6) and the absence of phase transitions for one-dimensional Hamiltonians with finite range interactions. Note that $\bar{H}(\eta) \equiv H(\bar{\psi}, \eta)$ is a one of these Hamiltonians and $f\left(\eta_{\mathbf{i}}\right)$ is a non-invariant quantity under the global symmetry group $G_{j}$ [see Eq.(2)], [18].

Corollary III. Any local quantity which is not gauge invariant under a two-dimensional continuous gauge group has a vanishing mean value at any finite temperature for systems with finite range interactions. This results from the combination of our theorem [Eq. [6] with the Mermin-Wagner theorem:

$$
\lim _{h \rightarrow 0} \lim _{N \rightarrow \infty} \frac{\sum_{\left\{\eta_{\mathbf{i}}\right\}} f\left(\eta_{\mathbf{i}}\right) e^{-\beta H(\bar{\psi}, \eta)-\beta h \sum_{\mathbf{i} \in \mathcal{C}_{j}} \eta_{\mathbf{i}}}}{z_{\{\bar{\psi}\}}}=0 .
$$

Where we have used that $G_{j}$ is a continuous symmetry group of $\bar{H}(\eta)=H(\bar{\psi}, \eta), f\left(\eta_{\mathbf{i}}\right)$ is a non-invariant quantity for $G_{j}$ 
[see Eq. [2] ], and $\bar{H}(\eta)$ is a two-dimensional Hamiltonian that only contains finite range interactions. [19]

The generalization of our theorem to the quantum case is very straightforward if we choose a basis of eigenvectors of the local operators that are linearly coupled to the symmetry breaking field $h$. In this basis, the states can be written as a direct product $|\phi\rangle=|\psi\rangle \otimes|\eta\rangle$. Eq. (6) is re-obtained with the sums replaced by traces over the states $|\eta\rangle$ :

$$
\left|\left\langle f\left(\phi_{\mathbf{i}}\right)\right\rangle_{h, N}\right| \leq\left|\frac{\operatorname{Tr}_{\left\{\eta_{\mathbf{i}}\right\}} f\left(\boldsymbol{\eta}_{\mathbf{i}}\right) e^{-\beta H(\bar{\psi}, \boldsymbol{\eta})-\beta h \sum_{\mathbf{i} \in \mathcal{C}_{j}} \boldsymbol{\eta}_{\mathbf{i}}}}{\operatorname{Tr}_{\left\{\eta_{\mathbf{i}}\right\}} e^{-\beta H(\bar{\psi}, \boldsymbol{\eta})-\beta h \sum_{\mathbf{i} \in \mathcal{C}_{j}} \boldsymbol{\eta}_{\mathbf{i}}}}\right|,
$$

In this case, $|\bar{\psi}\rangle$ corresponds to one particular state of the basis $|\psi\rangle$ that maximizes the right side of Eq. (8). Generalizing standard proofs, e.g. [20], we find in the quantum arena a zero temperature extension of Corollary III whenever there is a gap in the excitation spectrum:

Corollary IV. If a gap exists in a system possessing a $d \leq 2$ dimensional continuous symmetry (or, at least, an emergent continuous symmetry) in its low energy sector, then the expectation value of any local quantity which is not invariant under this symmetry, strictly vanishes at zero temperature.

\section{PHYSICAL REALIZATIONS AND IMPLICATIONS}

Armed with the above results, we briefly review known consequences of Elitzur's theorem and then focus at length on several of the applications of our new results.

\section{A. Local gauge symmetries- Pure and Matter coupled gauge theories and Spin glass systems}

a) Gauge theories- The application of Elitzur's theorem in gauge theories is well known and reviewed in excellent works such as [21]. No work on Elitzur's theorem is complete without a mention of its most prominent application. In theories of matter at lattice sites $\left(\sigma_{i}\right)$ coupled to gauge fields $\left(U_{i j}\right)$ residing on links, the action is a sum of a plaquette product of the gauge fields and (minimal) coupling between matter fields through the gauge [22]. To illustrate, consider the action in a $Z_{2}$ setting,

$$
S=-K \sum_{\square} U_{i j} U_{j k} U_{k l} U_{k l}-J \sum_{i j} \sigma_{i} U_{i j} \sigma_{j},
$$

with $U_{i j}= \pm 1$ and $\sigma_{i}= \pm 1$ elements of $Z_{2}$. Many pioneering results were found by [23]. The action is invariant under the local gauge transformation $\sigma_{i} \rightarrow \eta_{i} \sigma_{i}, U_{i j} \rightarrow \eta_{i} U_{i j} \eta_{j}$ with $\eta_{i}= \pm 1$ an element of $Z_{2}$. Defining the gauge invariant link variables $z_{i j} \equiv \sigma_{i} U_{i j} \sigma_{j}$ of which the action is a functional, we note that any correlator involving a product of any number of $z$ 's ("mesons") need not vanish. In the absence of coupling to matter $(J=0)$ - the "pure gauge theory"- only products of $U_{i j}$ along closed "Wilson" loops $\left(W=\left\langle\prod_{i j \in C} U_{i j}\right\rangle\right)$ are gauge invariant and may attain finite expectation values. b) Spin glasses- Perhaps one of the simplest realizations of spin-glasses is the Edwards-Anderson (EA) model [24] with

$$
H=-\sum_{\langle i j\rangle} J_{i j} \vec{S}_{i} \cdot \vec{S}_{j}-\vec{h} \cdot \sum_{i} \vec{S}_{i} .
$$

Here, $i$ and $j$ are nearest neighbor sites of a regular lattice and the three component spins $\vec{S}=\left(S_{x}, S_{y}, S_{z}\right)$ are considered as classical vectors. In the EA model, the exchange constants $J_{i j}$ are independent random variables drawn from a (Gaussian) probability distribution $P\left(J_{i j}\right)$. In spin glass systems, the experimentally measured expectation value of any observable corresponds to a quenched average over the distribution $P(J)$,

$$
\langle f(\{\vec{S}\})\rangle_{\text {quench }}=\int d J P(J)\langle f(\{\vec{S}\})\rangle_{J} .
$$

Here, $\langle f(\{\vec{S}\})\rangle_{J}$ denotes the expectation value of $f(\{\vec{S}\})$ for a given quenched distribution of exchange constants $\left\{J_{i j}\right\}$. The basic premise of spin glass systems is that the free energies (and their derivatives) are to be averaged via the distribution $P(J)$ to obtain the corresponding quenched quantities. The quenched expectation value of Eq. 111) is, for any even distribution $P(J)$, invariant under the following local $Z_{2}$ gauge transformation,

$$
\vec{S}_{i} \rightarrow \eta_{i} \vec{S}_{i}, J_{i j} \rightarrow \eta_{i} J_{i j} \eta_{j}
$$

with $\eta_{i}= \pm 1$ (an element of $Z_{2}$ ). [25] Performing the quenched disorder average of Eq. 111) over $J_{i j}$ is a central task in spin glass problems and ingenious schemes have been devised. Parisi showed that the quenched average may be done by introducing $n$ (with $n \rightarrow 0$ ) replicas of the spin field $\left\{\vec{S}_{i}^{a}\right\}_{a=1}^{n}$ at each site all coupling via the same $J_{i j}$ thus still satisfying the gauge transformation of Eq.(12) detailed above with a replica independent $\eta_{i}[26]$. Within the replica formulation, the average over $J_{i j}$ may be performed first leading to a functional of the replicated spin fields alone. To attain a finite expectation value, the quantity of interest must be gauge invariant. The lowest order quantity invariant under local transformation is [27], [28] $Q_{i}^{a b}=\left\langle\vec{S}_{i}^{a} \cdot \vec{S}_{i}^{b}\right\rangle$ (as both spin field suffer a factor of $\eta_{i}$ under the local gauge transformation, and $\eta_{i}^{2}=1$ ). In this manner, we see how the usual spin-glass overlap order parameters ( $Q_{i}^{a b}$ and their sums) are dictated by Elitzur's theorem for the n-replica action. c) Many other examples are found in numerous spin systems of current interest, For instance, Kitaev's [29] exact solution of a special spin system on a honeycomb lattice owes its existence to gaugelike local symmetries implemented via spin products around individual hexagons. When these local symmetries are fused with Elitzur's theorem, we find that on-site magnetization is precluded.

\section{B. Exact Higher-Dimensional Gauge-like symmetries: Orbital systems}

a) Spins- In 3d orbital systems on cubic lattices, each transition metal atom is surrounded by an octahedral cage of oxygens. Crystal field splitting lifts the degeneracy of the five $3 \mathrm{~d}$ 
orbitals of the transition metal to that of two higher energy $e_{g}$ levels (residing in the space spanned by the two orbital states $\left|d_{3 z^{2}-r^{2}}\right\rangle$ and $\left|d_{x^{2}-y^{2}}\right\rangle$ wherein the electronic wavefunctions point towards the surrounding oxygens with an associated high energy Coulomb penalty) and to three lower energy $t_{2 g}$ levels (spanned by the three states $\left|d_{x y}\right\rangle,\left|d_{x z}\right\rangle$ and $\left|d_{y z}\right\rangle$ in which the wave-functions point away from the surrounding oxygen atoms). Following [3], the latter three $t_{2 g}$ states will henceforth be denoted by $|X\rangle,|Y\rangle$, and $|Z\rangle$.

The super-exchange Kugel-Khomskii Hamiltonian (KK) [30] depicts a coupling between orbital and spin degrees of freedom in transition metal $3 \mathrm{~d}$ systems such as the $t_{2 g}$ titanate $\mathrm{LaTiO}_{3}$ and the vanadate $\mathrm{LaVO}_{3}$. As demonstrated by KK, in orbital systems the super-exchange Hamiltonian is anisotropic with a strong dependence on the orbital state. The overlap between the transition metal orbitals amongst themselves (and more notably via intermediate oxygen orbitals) strongly determines the strength (and viability if any) of the spin exchange interactions along the crystalline directions. In $e_{g}$ systems, the orbital component of the KK Hamiltonian exhibits, on a cubic lattice of size $L \times L \times L$, a discrete $\left[Z_{2}\right]^{3 L}(d=2)$ gauge-like symmetry corresponding to reflections of spins in entire planes about certain axis [8], [9]. As emphasized in [3], the three level $t_{2 g}$ version of the KK Hamiltonian, which is relevant to the titanates, prohibits direct hopping of electrons of a certain orbital flavor along one lattice direction. The key feature of the super-exchange Hamiltonian is that it prohibits hopping via intermediate oxygen $\mathrm{p}$ orbitals between any two electronic states of orbital flavor $\alpha(\alpha=X, Y$, or $Z)$ along the $\alpha$ axis of the cubic lattice (see Fig 1 .

In these materials the KK Hamiltonian [30] can be written as $H=H_{X}+H_{Y}+H_{Z}$ with

$$
H_{\alpha}=J \sum_{\langle i j\rangle \in \alpha} \sum_{\beta, \gamma \neq \alpha} \sum_{\sigma \eta} c_{i, \beta, \sigma}^{\dagger} c_{i, \gamma, \eta} c_{j, \gamma, \eta}^{\dagger} c_{j, \beta, \sigma} .
$$

In this Hamiltonian, $\langle i j\rangle \in \alpha$ is a nearest neighbor bond along the cubic lattice $\alpha$ axis, $c_{i \beta \sigma}^{\dagger}$ creates an electron at the cubic lattice site $i$ in a $\beta$ orbital state of spin $\sigma$.

As is evident from Eq.13, a uniform rotation of all spins, whose electronic orbital state is $|\alpha\rangle$, in any given plane $(P)$ orthogonal to the $\alpha$ axis [3]

$$
c_{i \alpha \sigma}^{\dagger}=\sum_{\eta} U_{\sigma, \eta}^{(P)} d_{i \alpha \eta}^{\dagger}
$$

with $\sigma, \eta$ the internal spin polarization directions, leaves the KK Hamiltonian invariant.

This leads to a conservation of the net spin of the electrons of orbital flavor $|\alpha\rangle$ in any plane orthogonal to the cubic $\alpha$ axis. This manifests itself via a continuous $S U(2)$ planar gauge group. The continuous planar rotation $\hat{O}_{P ; \alpha} \equiv$ $\left[\exp \left(i \vec{S}_{P}^{\alpha} \cdot \vec{\theta}_{P}^{\alpha}\right) / \hbar\right]$ is generated by $\vec{S}_{P}^{\alpha}=\sum_{i \in P} \vec{S}_{i}^{\alpha}$, the sum of all the spins $\vec{S}^{i, \alpha}$ in the orbital state $\alpha$ in any plane $P$ orthogonal to the direction $\alpha$, is a symmetry operation (see Fig 1 . Our theorem immediately prohibits a finite magnetization in these systems $\langle\vec{S}\rangle=0$ (Corollary III)- coinciding with the central result of [3]. We note that this symmetry does not prohibit the much more robust nematic spin ordering. For instance, in the presence of orbital ordering in the $|\alpha\rangle$ state, the order parameter $P \equiv\left\langle\vec{S}_{\vec{r}} \cdot \vec{S}_{\vec{r}+\hat{e}_{\eta}}\right\rangle$ along the $\eta=\mathrm{x}, \mathrm{y}$, or $\mathrm{z}$ axes with $\eta \neq \alpha$ need not vanish by our theorem. In real systems, the ideal mathematical model of KK with its perfect gaugelike symmetry and Elitzur theorem forbidden magnetization is likely corrupted by small effects (e.g. the hopping amplitude $t_{\perp}$ along the $\alpha$ axis does not identically vanish). Indeed finite temperature magnetization is seen in many of these compounds. Generically, in systems where the gauge-like symmetries inhibiting order are lightly lifted, we may naturally anticipate the now allowed ordering to be fragile and to exhibit a low transition temperature.

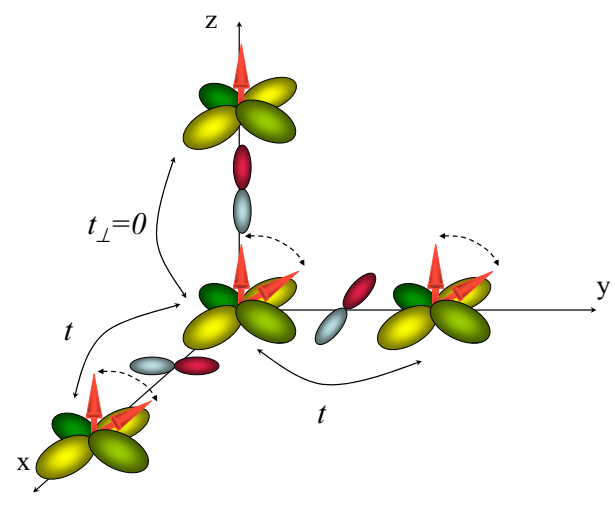

FIG. 1: (color online) The anisotropic hopping amplitudes leading to the Kugel-Khomskii (KK) Hamiltonian (Eq. 13]). Similar to [3], the four lobed states denote the $3 d$ orbitals of a transition metal while the intermediate small $p$ orbitals are oxygen orbital through which the super-exchange process occurs. The dark and bright shades denote positive and negative regions of the orbital wave-function. Due to orthogonality with the intermediate oxygen $p$ states, in any orbital state $|\alpha\rangle$ (e.g. $|Z\rangle \equiv\left|d_{x y}\right\rangle$ above), hopping is forbidden between two states separated along the cubic $\alpha$ ( $\mathrm{Z}$ above) axis. The ensuing super-exchange (KK) Hamiltonian exhibits a two-dimensional $S U(2)$ gauge symmetry corresponding to a uniform rotation of all spins whose orbital state is $|\alpha\rangle$ in any plane orthogonal to the cubic direction $\alpha$. In the above all spins whose orbital states are $|Z\rangle$ have been rotated in an arbitrary plane orthogonal to the cubic $\mathrm{Z}$ axis.

b) Orbitals- Orbitals in $3 \mathrm{~d}$ systems interact amongst themselves via Jahn-Teller distortions which for much the same reasons as in the KK Hamiltonian are anisotropic. The canonical prototype of all orbital interactions is the orbital compass model [31]. To streamline the essential physics in its simplest form, we examine the two-dimensional version of the orbital compass model. A complete mathematical analysis is detailed elsewhere [10]. In the two-dimensional orbital compass model at each site $\vec{r}$ of an $L \times L$ square lattice there is a $\mathrm{S}=1 / 2$ operator denoted, throughout for XY spins, by a lower case $\vec{s}_{\vec{r}}=\frac{\hbar}{2} \vec{\sigma}_{\vec{r}}$ (the upper case $\vec{S}$ will be reserved for three component spins). The planar orbital compass model Hamiltonian is:

$$
H=\frac{J}{4} \sum_{\vec{r}}\left(\sigma_{\vec{r}}^{x} \sigma_{\vec{r}+\hat{e}_{x}}^{x}+\sigma_{\vec{r}}^{y} \sigma_{\vec{r}+\hat{e}_{y}}^{y}\right) .
$$


In the three-dimensional model, additional nearest neighbor $\sigma^{z} \sigma^{z}$ bonds appear parallel to the z-axis. We will come to this in Eq. (21) later on. To tabulate the symmetries in this system, we define an operator on an arbitrary line $\mathrm{P}$ (of intercept y) $\hat{O}_{P ; y} \equiv \prod_{\vec{r} \in P} \sigma_{\vec{r}}^{y}$ with a similar definition for $\hat{O}_{P ; x}$. Up to global multiplicative phase factors, $\hat{O}_{P ; y}$ is a rotation by $\pi$ about the $\mathrm{x}$ axis of all sites $\vec{r}$ in the line $P$ whose $\mathrm{x}$ component is $r_{x}=x$. Similarly, the operator $\hat{O}_{P ; x}=\exp \left[i(\pi / 2) \sigma_{P}^{x}\right]$ with $\sigma_{P}^{x}=\sum_{\vec{r} \in P} \sigma_{\vec{r}}^{x}$. This is very much similar to the operators which appeared in our discussion above of the planar gauge-like symmetries of the KK model, yet for the orbitals here, these symmetries are discrete (the angle of rotation $\theta_{P}$ is quantized). From the products $\hat{O}_{P ; z}^{-1} \sigma_{\vec{r}}^{P ; x, y} \hat{O}_{P ; z}=-\sigma_{\vec{r}}^{x, y}$, it is clear that $\hat{O}_{P ; z}$ is a symmetry of $H$. Similarly, $\hat{O}_{P ; x}$ inverts the y-component of all spins on the line of intercept $x$ while leaving $\sigma^{x}$ untouched. In arbitrary spin size (S) versions of this model, the string operators above containing spin-1/2 Pauli matrices may be replaced by the corresponding spin-S $\pi$-rotations of all spins in the line $P$. These "string" operators spanning spin operators on entire lines commute with the Hamiltonian, $\left[H, \hat{O}_{P ; \alpha}\right]=0$. As in the case of spins in orbital $t_{2 g}$ systems, rotations of individual lower-dimensional "planes" (lines) about an axis orthogonal to them leave the system invariant. As a consequence of these symmetries, each state is, at least, $\mathcal{O}\left(2^{2 L}\right)$ degenerate.

As there exist one-dimensional gauge-like symmetries $\hat{O}_{P ; x, y}$ in this system, by corollary (ii) the magnetization vanishes identically and only quantities invariant under $\hat{O}_{P ; x, y}$ may attain a finite expectation value. Indeed, it has been established that a nematic-type order respecting the gauge-like symmetries exists in related classical extensions of this system [7, 8, 9]. More sophisticated order parameters $\langle T\rangle$ (invariant under $\left\{\hat{O}_{P: x}, y\right\}$ ) and their composites may be trivially constructed [10]. In these, $\langle T\rangle \equiv\left\langle\prod \sigma_{\vec{r}}^{x} \sigma_{\vec{r}^{\prime}}^{y}\right\rangle$, the number of $\left\{\sigma_{\vec{r}}^{x}\right\}$ on any given horizontal row and the number of $\left\{\sigma_{\vec{r}^{\prime}}^{y}\right\}$ on any given vertical column are both even. Due to the anti-commuting nature of $\sigma_{\vec{r}}^{x}$ and $\sigma_{\vec{r}}^{z}$, any specific operator $T_{i}$ commutes with all $2 L$ inversion operators $\left\{\hat{O}_{P ; x, y}\right\}$, with the Hamiltonian $H$ and with any other operator $T_{j \neq i}$ [10]. Obviously, the largest and most physically relevant order parameter is the minimal gauge invariant dimer like nematic order parameters $\left\langle S_{i}^{\alpha} S_{i+\hat{e}_{\alpha}}^{\alpha}\right\rangle$ (with $\alpha=x, y$ ), and their linear combinations.

For the three-dimensional " $120^{\circ}$ model" encapsulating the Jahn-Teller distortions and the orbital only component of the orbital-spin KK Hamiltonian in " $e_{g}$ " orbital systems (such as e.g. the colossal magneto-resistive manganate $\mathrm{LaMnO}_{3}$, the cuprate $\mathrm{KCuF}_{3}$, the nickelate $\mathrm{LiNiO}_{2}, \mathrm{Rb}_{2} \mathrm{CrCl}_{4}$, and many other systems) novel discrete planar gauge-like symmetries and a finite on-site magnetization were found [8],[9]. The three-dimensional " $120^{\circ}$ model" model possesses an exact discrete $(d=2)\left[Z_{2}\right]^{3 L}$ gauge-like symmetry (by sequential planar Rubick's cube like reflections about internal spin directions; See Fig.1 in Refs. [8],[9] for an illustration). Unlike the case for the spins in the KK Hamiltonian for orbital $t_{2 g}$ systems, there are no exact nor emergent continuous gaugelike symmetries. In accord with our postulates, the three- dimensional $120^{\circ}$ model can (and indeed does) have a finite magnetization [8], [9].

\section{Exact Gauge-Like Symmetries: Superconducting Arrays}

A related system having only four-spin interactions (i.e. with absolutely no two spin interactions), which emulates $(p+$ $i p$ ) superconducting grains (such as those of e.g. $\mathrm{Sr}_{2} \mathrm{RuO}_{4}$ ) on a square grid, was recently investigated by $\mathrm{Xu}$ and Moore [4], [5]. The Hamiltonian of this system is

$$
H=-K \sum_{\square} \sigma^{z} \sigma^{z} \sigma^{z} \sigma^{z}-h \sum_{\mathbf{r}} \sigma_{\mathbf{r}}^{x}
$$

Here, the four spin product is the product of all spins common to a given plaquette $\square$. Note that the spins reside on the vertices on the plaquette (not on its bonds as gauge fields). It has been established via duality mappings [10], that this model of superconducting arrays is identical to the two-dimensional orbital compass model discussed in IIIBb). Not too surprisingly, the systems investigated by $\mathrm{Xu}$ and Moore have clear one-dimensional gauge-like symmetries similar to those of the two-dimensional orbital compass discussed in IIIBb). Rather explicitly,

$$
\hat{O}_{P}=\prod_{\vec{r} \in P} \sigma_{\vec{r}}^{x}
$$

with $P$ any horizontal row or vertical column, is a symmetry of Eq.16. These symmetries are trivially related to the gauge-like symmetries of the planar orbital compass model upon invoking the duality mappings of [10].

\section{Other systems with Gauge-Like Symmetries}

Many other pioneering works unveiled plenty of new gauge-like symmetries in other systems. In [13], it was shown that ring exchange Bose metals, in the absence of nearest neighbor Boson hopping, exhibit much the same onedimensional gauge-like symmetries discussed above. Similar sliding symmetries of Hamiltonians (actions) invariant under arbitrary continuous deformations of a certain field along a transverse direction,

$$
\phi(x, y) \rightarrow \phi(x, y)+f(y)
$$

appear in many systems. As much throughout this work, in these systems there exists no rigidity to shear deformations along one (or more) direction(s) ( $y$ in Eq. (18). Many such systems were discovered and discussed at length in rather novel works on Quantum Hall liquid crystalline phases [14], [15], a number of models of lipid bilayers with intercalated DNA strands [16], and "Luttinger Liquid Arrays" or "sliding Luttinger liquids" [17] which, as suggested in various works, may be intimately related to "stripes" [32], [33]. The consequences of our theorem (and Corollaries II, III in particular) are immediate for these systems, $\langle\phi\rangle=0$. 


\section{E. Exact Gauge-Like Symmetries by Inverted Dimensional Reductions}

Many spin systems can be systematically generated to have the gauge-like symmetries of orbital systems (as well as many others) [6]. We found a very simple algorithm doing so which very lucidly invokes dimensional reduction in the reverse order. The basic idea underlying our scheme is that a highdimensional system containing decoupled chains or planes is, of course, one-dimensional and possesses trivial one- or two-dimensional gauge-like symmetries. To illustrate, we first consider a one-dimensional XY spin chain having nearest neighbor interactions,

$$
H=J \sum_{\langle i j\rangle} \vec{s}_{i} \cdot \vec{s}_{j}
$$

with $i$ and $j$ nearest neighbor lattice sites and $\vec{s}=\left(s_{x}, s_{y}\right)$ an $\mathrm{XY}$ spin. This translationally invariant Hamiltonian may, of course, be expressed in Fourier space,

$$
H=\frac{1}{2 N} \sum_{\vec{k}} s_{\alpha}(\vec{k}) v_{\alpha \beta}(\vec{k}) s_{\beta}(-\vec{k}),
$$

where $\alpha$ and $\beta$ denote the two internal spin polarization directions, and the matrix $v_{\alpha \beta}=\left(2 J \delta_{\alpha \beta} \cos k\right)$. Let us now make this one-dimensional system higher-dimensional by replacing the scalar quantity $k$ by more complicated quantities. If we replace the scalar momentum $k$ of the one-dimensional spin chain by a two-dimensional $\vec{k}$ which appears in $v(\vec{k})$ only via $[\cos (\vec{k} \cdot \vec{a})]$ with $\vec{a}$ a real vector, then the twodimensional system will be a trivial realization of decoupled chains (interacting along the direction $\hat{a}$ ) and trivially possessing one-dimensional gauge symmetries. We may now replace the c-number coefficients $a_{i}$ by operators to obtain non-trivial systems. Some of the simplest examples are afforded when $\left\{a_{i}\right\}$ are chosen to be projection operators. For instance, by replacing the scalar $k$ of the one-dimensional spin chain by a simple two-dimensional diagonal matrix, we obtain $v(\vec{k})=2 J \mathrm{dg}\left(\cos k_{x}, \cos k_{y}\right)$. (Here and throughout, $\operatorname{dg}\left(a_{1}, a_{2}, \ldots, a_{d}\right)$ refers to a $d$-dimensional diagonal whose diagonal elements are $\left.\left\{a_{1}, a_{2}, \ldots, a_{d}\right\}\right)$. In the $v(\vec{k})$ above, we replicate the single momentum coordinate to generate a higher-dimensional interaction. Insertion of this matrix into Eq. 20, leads to the two-dimensional orbital compass model of $\mathbf{B b}$ ) having $\left[Z_{2}\right]^{2 L}$ gauge-like symmetries of "en-block" rotations by $\pi$ of all spins belonging to a certain row/column.

Similarly, by setting $v(\vec{k})=2 J \mathrm{dg}\left(\cos k_{x}, \cos k_{y}, \cos k_{z}\right)$ in Eq. (20) for a three component spin system $\vec{S}=$ $\left(S_{x}, S_{y}, S_{x z}\right)$, we obtain the three-dimensional orbital compass model (on a cubic lattice of size $L \times L \times L$ )

$$
H=J \sum_{i}\left(S_{i}^{x} S_{i+\hat{e}_{x}}^{x}+S_{i}^{y} S_{i+\hat{e}_{y}}^{y}+S_{i}^{z} S_{i+\hat{e}_{z}}^{z}\right) .
$$

This system has an exact $\left[Z_{2}\right]^{3 L^{2}}$ symmetry for classical spins (along each chain parallel to the cubic $\alpha$ axis, we may reflect $S_{\alpha} \rightarrow-S_{\alpha}$ yet keep all other spin polarizations unchanged,
$\left.S_{\beta \neq \alpha} \rightarrow S_{\beta \neq \alpha}\right)$ as well as a lower exact $\left[Z_{2}\right]^{3 L}$ gauge-like symmetry (forming a subset of the larger $\left[Z_{2}\right]^{3 L^{2}}$ symmetry present for classical spins) when the spins are quantum: in this case we may rotate all spins in a plane orthogonal to the cubic lattice direction $\alpha$ by $\pi$ about the internal $S_{\alpha}$ quantization axis.

In Eq. 20 for an XY spin system, we may further generate interactions along arbitrary tilted rays by considering $v=2 J \cos \left(k_{x}+\lambda k_{y} \sigma_{3}\right)$ with $\sigma_{3}$ the Pauli matrix. An insertion this kernel into Eq. 20 leads to real space interactions of the $\mathrm{x}$ components of the spins along chains parallel to the $(1, \lambda)$ direction and of an exchange interaction amongst the $y$ components of the spins along the $(1,-\lambda)$ direction. By tuning the $\lambda \rightarrow 0$, the two "clapping" $(1, \pm \lambda)$ directions fold back and degenerate into the original one-dimensional XY spin chain. We find that for all $\lambda \neq 0$, the classical symmetries are much the same as for the two-dimensional orbital compass model: reflecting all $s_{x} \rightarrow-s_{x}$ while leaving $s_{y}$ untouched along any chain parallel to $(1, \lambda)$. Thus, according to our theorem and corollary (II), no finite on-site magnetization is possible in classical XY systems with the above Hamiltonian. Nematic like order is possible and was indeed found [7] for $\lambda= \pm 1$. For $\lambda \neq \pm 1$, there is, however, no canonical transformation that may be applied to the quantum spin model to encapsulate these symmetries.

If we start with a nearest neighbor three component (Heisenberg) spin model on the square lattice and replace the usual isotropic interaction kernel $2 J \mathrm{dg}\left(\cos k_{x}+\right.$ $\left.\cos k_{y}, \cos k_{x}+\cos k_{y}\right)$ by $v=\operatorname{dg}\left(\cos k_{x}+\cos k_{y}, \cos k_{x}+\right.$ $\left.\cos k_{y}, \cos k_{z}\right)$ to generate a spin system residing on a cubic lattice, we obtain a new system with many exact gauge-like symmetries. In classical systems, for all chains parallel to the z-axis we may take $S_{z} \rightarrow-S_{z}$ and for all planes parallel to the XY plane we may perform a uniform continuous rotation about the $\mathrm{z}$ axis- a $[U(1)]^{L} \times Z_{2}^{L^{2}}$ symmetry. If the spins are quantum, only the canonical $[U(1)]^{L}$ symmetries are present. Our theorem precludes finite on-site order for both classical and quantum spins on a cubic lattice with the above spin Hamiltonian.

We may similarly start with many other lower-dimensional systems to generate higher-dimensional systems by these operations. In some instances, these transformations lead to systems of intense physical interest (e.g. orbital compass models employed for elucidating the properties $3 \mathrm{~d}$ transition metal compounds at large [31], and crystal field split $t_{2 g}$ systems in particular). For each of these systems, our theorem and its corollaries can be applied to often inhibit the possibility of a finite on-site magnetization. It should be emphasized that although these models are generated by artificially "lifting" a low-dimensional momentum, the resulting Hamiltonians are, obviously, not lower dimensional. We are only considering symmetry properties here and not the thermodynamics. In the current context, "dimensional reduction" may be understood from a technical point of view as an algorithm for yielding gauge-like symmetries in a natural fashion. Thermodynamic dimensional reduction of these models occurs only in the large $n$ (spherical) limit of classical renditions of all of the above mentioned spin systems (e.g. the two-dimensional compass 
model is identical to an XY chain). We will briefly comment to other special properties of large $n$ systems when discussing new emergent gauge-like symmetries that we found in Brazovskii-type systems relevant, amongst other things, to liquid crystal systems, theories of structural glasses, electronic systems favoring phase separation that is inhibited by long range Coulomb interactions, and to many other arenas.

By reverse engineering various given gauge-like lattice symmetries to find corresponding Hamiltonians, we found additional models on different lattices having gauge-like symmetries when polarization (spin space) dependent spin-spin interactions were slaved to the bond directional on the lattice (external space) to produce many different real space interaction kernels $v_{\alpha \beta}(\vec{k})$ (or their direct real space transforms $\left.V_{\alpha \beta}\left(\vec{r}_{i}, \vec{r}_{j}\right)\right)$.

\section{F. Emergent gauge-like symmetries in spin, orbital, and other systems}

We now examine situations wherein a gauge-like symmetry emerges within the low energy sector. In some instances, it is present in leading order fluctuations about the classical ground state (e.g. 1/S spin wave corrections). From corollary (IV), we know that in the presence of a gap, expectation values of any symmetry non-invariant quantities must vanish. In general systems having various emergent gauge-like symmetries, non-gauge invariant quantities may often vanish. I. Quantum systems: a) Geometrically Frustrated Spin systems: In many spin systems (e.g. the kagome, pyrochlore, and checkerboard magnets), the availability of many spins vis a vis the number of building blocks on which they reside allows the proliferation of many low energy states [34]. Pyrochlore magnets such as the $\mathrm{S}=3 / 2$ spinel $\mathrm{ZnCr}_{2} \mathrm{O}_{4}$ have remarkable magnetic behavior (such as e.g. very clearly detectable spin waves linking degenerate states). The checkerboard lattice [35] may be viewed as a two-dimensional projection of the three dimensional pyrochlore lattice composed of corner sharing tetrahedra on the plane. The system is composed of nearest neighbor bonds on the square lattice augmented by additional diagonal, next nearest neighbor, interactions along all diagonals of one plaquette sub-lattice (hence the name "checkerboard"). Both the pyrochlore and checkerboard magnets has an exponential in volume classical ground state degeneracy as in other systems of emergent local (or $d=0$ ) gauge symmetry. Leading order $(1 / S)$ corrections were computed finding the effective spin-wave Hamiltonian for fluctuations about the ground states [35, 36]. Much like the orbital systems discussed in Section (IIIBb), the resulting Hamiltonian possesses one dimensional gauge like symmetries. In [37, an effective low energy Hamiltonian was derived for these quantum systems without the need for spin-wave theory. The resulting effective Hamiltonian looks much the same as the KK Hamiltonian for orbital systems discussed earlier and displays much the same discrete gauge-like symmetries seen in the KK relevant to ordering in $e_{g}$ materials [8],[9]. Gauge-like theories for geometrically frustrated magnets with string operators generating freely propagating "spinons" are also found elsewhere, e.g.
[38] for the pyrochlore magnet. The large $S$ ground states of kagome antiferromagnets may be linked to each other via all possible contortions of a two-dimensional membrane. The effective low energy Hamiltonian of the quantum Kagome magnet is, once again, similar to the KK Hamiltonian. [39]

b) Spin systems with ring exchange: In a recent work [12], emergent one dimensional gauge like symmetries were found in a quasi-exactly solvable square lattice spin system of a Heisenberg antiferromagnet augmented by ring exchange interactions of comparable strength. Here, the presence of onedimensional gauge like symmetries went hand in hand with fractionalization: spinons propagating along the diagonal directions incurred no energy penalty while spin motion in other directions led to a confining potential. In an upcoming work [40], we identify gauge-invariant order parameters in this system and further suggest the existence of a finite temperature "order out disorder" transition suggesting natural finite temperature fractionalization in these two-dimensional systems.

II. Classical spin systems: a) Within the classical (large S) limit of $\mathrm{d}=3$ orbital compass of Eq.21) (relevant to such compounds as $\mathrm{YVO}_{3} \mathrm{LaVO}_{3}$, and $\mathrm{LaTiO}_{3}$ ), we find an emergent continuous rotational symmetry of all the spins in an entire plane about a spin axis orthogonal to the plane direction. For instance, taking any uniform spin state (automatically a ground state of Eq.21) and rotating all of the spins in a given plane orthogonal to the z-axis (i.e. all spins on sites $\vec{r}$ satisfying $r_{z}=z$ ) by an arbitrary angle $\theta$ about the $S_{z}$ spin polarization axis leads to another ground state. This can be further mutated by similar operations in other planes (parallel and orthogonal). Compounding all of the symmetries together, this corresponds to an emergent $[U(1)]^{3 L}$ degeneracy. Indeed, it was found ([9]) that the system has nematic like order invariant under this continuous planar gauge-like symmetry. b) The Fully Frustrated XY model on a honeycomb lattice: within the ground state of such an XY system having half a fluxon threading each hexagonal "plaquette" [41], each "plaquette" has exactly two bonds having zero phase gradient (similar to the requirement of one singlet per square plaquette in [12]) with all other bonds having a phase gradients of $\pi / 4$. Connecting the zero gradient bonds to each other leads to nonintersecting stripes threading the system. There are several ways of generating such stripe configurations, all of which are inter-related by an emergent one dimensional like gauge symmetry. c) An Ising spin systems on a square lattice with competing next and next nearest neighbor [42] interactions, having a shell of minimizing modes in Fourier space, was found to possess an one-dimensional emergent gauge-like symmetry with a diagonal stripe like structure within its ground state. d) General two spin Fourier kernels, such as $v(\vec{k})=\left(k^{2}-q^{2}\right)^{2}$ in Eq. 20), with a $(d-1)$ dimensional shell of minimizing modes in Fourier space are relevant amongst other things to the Brazovskii models [43] for liquid crystals, block copolymers, frustrated electronic systems, and structural glass transitions [44, 45]. We may rigorously establish, in the large $n$ (spherical) limit, that the ground state entropy scales as the surface area of the system, $S \propto(q N)^{(d-1) / d}$ [46]. The ground state possess an $S O\left(N^{(d-1) / d}\right)$ symmetry of which a gauge-like $[U(1)]^{\frac{N^{(d-1) / d}}{2}}$ is a subgroup. The Brazovskii sys- 
tem is endowed with a continuous symmetry harboring a one dimensional gauge-like character. Similar to our corollary for gapped quantum systems, in the large $n$ Brazovskii system the on-site magnetization is zero and, in fact, the system possesses a zero temperature critical point. Indeed in all translationally invariant systems with a kernel $v(\vec{k})$ analytic about its minima and in which the minimizing Fourier modes form a $(d-1)$ dimensional manifold (as in rotationally invariant incommensurate systems) or a $(d-2)$ dimensional manifold corresponding to continuous one or two-dimensional gauge symmetries. In large $n$ spin systems, any permutation of the values of the kernel $v(\vec{k}) \rightarrow v(P \vec{k})$ (with $P$ any permutation of the $N$ momenta) leaves the partition function identically invariant [47]. This allows an exact reduction to decoupled chains at low temperatures where gauge-like symmetries trivially exist. Such symmetries were also shown to rigorously exist in large $n$ renditions of vectorial spins when subjected to uniform non-Abelian gauge backgrounds. [45] [Within the spherical model, the inverse critical temperature is given by

$$
\frac{1}{k_{B} T_{c}}=\int \frac{d^{d} k}{(2 \pi)^{d}} \frac{1}{v(\vec{k})-v(\vec{q})}
$$

which diverges for $(d-1)$ or $(d-2)$ dimensional manifolds of modes $\vec{q}$ which minimize an analytic kernel $v(\vec{k})$.] These symmetries lead to "zero-energy domain walls" which go hand in hand with fractionalization in the quantum models of [12]. These high degeneracies do not generically survive for finite $n$ system (where, for example, XY spin systems with the same kernel have an emergent discrete $Z_{M}$ symmetry (with $\left.\left.M \sim(q L)^{d-1}\right)\right)$. Nevertheless, in some special instances they do, e.g. [42].

\section{DISCUSSION}

The degeneracy found in all (or only ground) states in systems having exact (or, respectively, only emergent) gauge-like symmetries suggests deep non-trivialities in the ordering transition that occurs, if any, at low temperatures.

As well known, in the presence of ground state degeneracies, entropic fluctuations often drive the system to order in the vicinity of "softer" states having more phase space for low energy fluctuations about them [34]. This mechanism is often termed "Order out of Disorder". The origin of this name are the entropic fluctuations ("disorder") which drive the low temperature ordering transition. The presence of gaugelike symmetries makes many standard methods of analysis much harder or inexact. Nevertheless, finite temperature orbital ordering (via an "order out of disorder" mechanism) was rigorously established in [8], [9] for two classical systems having gauge-like symmetries. Finite temperature magnetization was proved in the 120 degree model which harbors an exact $\left[Z_{2}\right]^{3 L}$ symmetry. Nematic order was also shown to rigorously exist in the classical three dimensional orbital compass model which has an exact $\left[Z_{2}\right]^{3 L}$ symmetry and an emergent $[U(1)]^{3 L}$ symmetry. The techniques presented in [8], [9] may be straightforwardly applied to a host of other systems possessing gauge-like symmetries, e.g. the effective low energy Hamiltonians of [37], [39] for the pyrochlore and kagome magnets. The natural candidates for order parameters are quantities invariant under all low dimensional gauge-like symmetries (exact or emergent). With this guiding principle in hand, a natural order parameter is found for two-dimensional quantum spin systems [12] exhibiting finite temperature fractionalization. [40]

In summary, we showed that the generalization of Elitzur's theorem to $d$-dimensional gauge groups (intermediate between local and global gauge symmetries) provides a formal meaning for the notion of dimensional reduction. Our theorem proves that for quantities which are non-invariant under the $d$-dimensional gauge transformations, the effective dimension of $D$-dimensional theory under consideration is reduced from $D$ to $d$. Elitzur's theorem is then a consequence of the absence of phase transitions at any finite temperature in a zero-dimensional system (see our corollary I). Moreover, this result also implies that a one-dimensional gauge symmetry cannot be broken if the interactions of the theory have a finite range (corollary II). The same holds true for continuous two-dimensional gauge symmetries (corollary III). We further described different theories of current interest which possess exact gauge-like symmetries for which corollaries II and III directly apply. Additional systems are known to have emergent gauge like symmetries and we illustrated (corollary IV) how Elitzur's theorem extends even to such systems in the presence of gaps. The wealth of systems of current interest for which our theorem and its four corollaries directly apply illustrates the power and generality of our result.

This work was sponsored by the US DOE under contracts W-7405-ENG-36, PICT 03-06343 of ANPCyT and LDRD X1WX. ZN gratefully acknowledges E. Fradkin and a productive brief formal visit to UIUC which further sparked [10].
[1] N. D. Mermin and H. Wagner, Phys. Rev. Lett. 17, 1133 (1966).

[2] S. Elitzur, Phys. Rev. D 12, 3978 (1975).

[3] A. B. Harris, T. Yildirim,A. Aharony, O. Entin-Wohlman and I. Y. Korenblit, Phys. Rev. Lett. 91, 087206 (2003).

[4] C. Xu and J. E. Moore, Phys. Rev. Lett. 93, 047003 (2004).

[5] C. Xu and J. E. Moore, cond-mat/0405271

[6] Z. Nussinov, cond-mat/0103188

[7] A. Mishra et al, cond-mat/0407470

[8] Z. Nussinov, M. Biskup, L. Chayes, J. v. d. Brink, Europhysics
Letters, 67, 990-996 (2004)

[9] M. Biskup, L. Chayes, Z. Nussinov, cond-mat/0309691 Communications in Math. Phys., To appear; ibid, "Ordering in Transition Metal Oxides: II. The Orbital Compass Model”, in preparation

[10] Z. Nussinov and E. Fradkin, cond-mat/0410720 Phys. Rev. B, To appear

[11] C. D. Batista and G. Ortiz, Adv.in Phys. 53, 1 (2004).

[12] C. D. Batista and S. A. Trugman, Phys. Rev. Lett. 93, 217202 
(2004).

[13] A. Paramekanti, L. Balents, and M. P. A. Fisher, Phys. Rev. B 66, 054526 (2002)

[14] M. J. Lawler and E. Fradkin, cond-mat/0405237

[15] L. Radzihovsky and A. T. Dorsey, Phys. Rev. Lett., 88, 216802 (2002)

[16] C. S. O’Hern and T. C. Lubensky, Phys. Rev. E 58, 5948 (1998)

[17] V. J. Emery, E. Fradkin, S. A. Kivelson, and T. C. Lubensky, Phys. Rev. Lett., 85, 2160 (2000)

[18] For readers wishing to have a simple concrete example in mind for which the physical underpinning can be made transparent, we now sketch matters for the two-dimensional orbital compass model. Here, the Hamiltonian is $H=J \sum_{i}\left(S_{i}^{x} S_{i+\hat{e}_{x}}^{x}+\right.$ $\left.S_{i}^{y} S_{i+\hat{e}_{y}}^{y}\right)$. Insofar as the energetics of spins on any row $\left(\mathcal{C}_{l}\right)$ parallel to the $x$ axis is concerned, the $y$ components of the spins on nearest neighbor rows act as external magnetic fields along the $S_{y}$ axis leading to the effective Hamiltonian, $H=$ $J \sum_{i} S_{i}^{x} S_{i+\hat{e}_{x}}^{x}+B_{i} S_{i}^{y}$, with $B_{i}=J\left(S_{i-\hat{e}_{y}}^{y}+S_{i+\hat{e}_{y}}^{y}\right)$. Here the fields $\{\eta\}$ of the main text are spin components along the row $\mathcal{C}_{l}$ and $\{\psi\}$ includes spins on all other rows in the system. For a certain choice of the spins on all other rows $\bar{\psi}$, we find the Hamiltonian which maximizes the expectation value $\left\langle S_{i}^{x}\right\rangle$ as read off from the square brackets in Eq. (4), $H(\bar{\psi}, \eta) \equiv \bar{H}(\eta)$. This corresponds to certain external fields $\left\{\bar{B}_{i}\right\}$ at the various lattice sites $i$. We now discern the viability of finite temperature discrete $Z_{2}$ symmetry breaking $\left(S_{x} \rightarrow-S_{x}\right)$ in this system. As well appreciated, in the absence of an external magnetic field along the y-axis $\left(\bar{B}_{i}=0\right)$, the one dimensional (Ising) system would not break $Z_{2}$ symmetry as a consequence of entropic effects overwhelming energetic domain wall penalties at all finite temperatures. This mismatch becomes even more acute in the presence of finite fields $\left\{\bar{B}_{i}\right\}$ along the y-axis as the spins become more canted away from the $\mathrm{x}$-axis, wherein the domain wall energies are even lower than in a simple one dimensional Ising model. This reduction in the energy penalty associated with symmetry breaking events vis a vis the corresponding energy penalties in low dimensional systems- which already would not order on their own right- is the physical engine behind our corollaries.

[19] To prove this explicitly, we follow the procedure of Mermin and Wagner and choose the quantities appearing in the Bogoliubov inequality appropriately. As an example, let us consider the Hamiltonian $\bar{H}=\frac{1}{2} \sum J_{i j}\left(S_{i}^{x} S_{j}^{x}+S_{y}^{i} S_{y}^{j}\right)-\sum_{i} \bar{B}_{i} S_{i}^{z}-$ $h S_{\vec{q}}^{x}$ where the infinitesimal external field couples to a given Fourier component $(\vec{q})$ of $S^{x}$. For $J_{i j}=J \delta_{|i-j|, 1}$, this Hamiltonian depicts an xy plane in the three dimensional orbital compass model (Eq. 21), (with a specific $\bar{B}_{i} \equiv\left(S_{i+\hat{e}_{z}}^{z}+\right.$ $\left.S_{i-\hat{e}_{z}}^{z}\right)$ maximizing the square brackets in Eq.(4). We now set, in the Bogoliubov inequality $\left|\left\langle\left[C^{\dagger}, A^{\dagger}\right]\right\rangle\right|^{2} \leq \frac{1}{2 T}\left(A A^{\dagger}+\right.$ $\left.A^{\dagger} A\right)\left\langle\left[C^{\dagger},[\bar{H}, C]\right]\right\rangle$, the quantities to be $C=S_{\vec{k}}^{z}$ (the Fourier component conjugate to the external $\bar{\psi}$ ) and $A=S_{-\vec{k}-\vec{q}}^{x}$ (corresponding to $\eta$ ). In the thermodynamic limit (wherein the number of lattices sites $N \rightarrow \infty$ ), this leads to a vanishing $m_{\vec{q}}=N^{-1}\left\langle S_{\vec{q}}^{x}\right\rangle$ at all finite temperatures when the external field applied $h \rightarrow 0$ in a system of short range interactions such that $\frac{1}{2 N} \sum_{i j} J_{i j}\left|\vec{x}_{i}-\vec{x}_{j}\right|^{2}$ (where $\vec{x}_{i}$ is the spatial location of site $i$ ) is bounded. Here, the external "magnetic fields" $\bar{B}_{i}$ acting along the z-axis do not spoil the invariance of the Hamiltonian under the symmetry group $G_{j}$ (corresponding to $O(2)$ rotations in the xy plane).

[20] Assa Auerbach, "Interacting Electrons and Quantum Magnetism", Springer-Verlang 1994 (chapter 6, in particular)
[21] J. B. Kogut, Reviews of Modern Physics, 51, 659 (1979)

[22] F. Wegner, J. Math. Phys. 12, 2259 (1971)

[23] E. Fradkin and S. H. Shenker, Phys. Rev. D 19, 3682 (1979)

[24] S. F. Edwards and P. W. Anderson, J. Phys. F 5, 89 (1979)

[25] D. C. Mattis, Phys. Lett. 56, 421 (1976). A local gauge transformation similar to that of Eq. 12 was first elucidated in a different context by Mattis. These unfrustrated Mattis spin glasses may all be related to a ferromagnet by the transformations of Eq. 12. In the current publication, we discuss extensions of such symmetries and their implications in general, far more frustrated, spin glass systems (such as the EA model of Eq. 10 ) in which the quenched average is explicitly computed.

[26] The central idea of the replica formulation is that the free energy averaged over in the quenched expectation value is trivially given by the identity $\ln Z=\lim _{n \rightarrow 0} \frac{Z^{n}-1}{n}$ with $Z$ the partition function and $n$ the number of replicas.

[27] G. Parisi, Phys. Rev. Lett., 50, 1946 (1983)

[28] G. Parisi, cond-mat/9411111]

[29] A. Kitaev, Unpublished.

[30] K. I. Kugel and D. I. Khomskii, Sov. Phys. JETP 37, 725 (1973); Sov. Phys. Usp. 25, 231 (1982)

[31] J. van den Brink, G. Khaliullin and D. Khomskii, Orbital effects in manganites, In: T. Chatterij (ed.), Colossal Magnetoresistive Manganites, Kluwer Academic Publishers, Dordrecht, 2002; cond-mat/0206053

[32] J. Zaanen and O. Gunnarsson, Phys. Rev. B 40, 7391 (1989); K. Machida, Physica C 158, 192 (1989); H. J. Schulz, Phys. Rev. Lett. 64, 1445 (1990)

[33] V. J. Emery and S. A. Kivelson, Physica C, 26, 44 (1996); U. Low, V. J. Emery,K. Fabricius, S. A. Kivelson, Phys. Rev. Lett. 72, 1918 (1994)

[34] R. Moessner, Can. J. Phys. 79, 1283 (2001), and references therein

[35] O. Tchernyshyov, O. A. Starykh, R. Moessner, and A. G. Abanov, Phys. Rev. B 68, 144422 (2003)

[36] C. L. Henley, unpublished

[37] E. Berg, E. Altman, and Assa Auerbach, Phys. Rev. Lett., 90, 147204 (2003)

[38] M. Hermele, M. P. A. Fisher, and L. Balents, Phys. Rev. B 69, 064404 (2004)

[39] R. Budnik and Assa Auerbach, cond-mat/0406651

[40] C. D. Batista and Z. Nussinov, in preparation

[41] S. E. Korshunov and B. Doucot, cond-mat/0310536

[42] Z. Nussinov, cond-mat/0105253

[43] S. A. Brazovskii, Sov. Phys.- JETP, 41, 85 (1975)

[44] G. H. Fredrickson and K. Binder, J. Chem. Phys. 91, 7265 (1989); J. Schmalian and P. G. Wolynes, Phys. Rev. Lett. 85 , 836 (2000); H. Westfahl, Jr., J. Schmalian, and P. G. Wolynes, Phys. Rev. B 64, 174203 (2001); K. K. Loh, K. Kawasaki, A. R. Bishop, T.Lookman, A. Saxena, Z. Nussinov and J. Schmalian, Phys. Rev. E, 69, 10501 (2004); D. Kivelson, S. A. Kivelson, X. Zhao, Z. Nussinov, and G. Tarjus, Physica A 219, 27 (1995); L. Chayes, V. J. Emery, S. A. Kivelson, Z. Nussinov, and G. Tarjus, Physica A 225, 129 (1996); T. Ohta and K. Kawasaki, Macromolecules 19, 2621 (1986); V. J. Emery and S. A. Kivelson, Physica C 209, 597 (1993); R. Choksi, J. Nonlinear Sci, 11, 223 (2001); M. Seul and D. Andelman, Science 267, 476 (1995)

[45] Z. Nussinov, Phys. Rev. B 69, 014208 (2004)

[46] The general ground state is given by the real space $\operatorname{spin} S\left(\vec{r}_{i}\right)=$ $\sum_{m} a_{q_{m}} \cos \left(\vec{q}_{m} \cdot \vec{r}_{i}+\phi_{m}\right)$ (with $\left\{\vec{q}_{m}\right\}$ the set of modes minimizing the interaction kernel $v(\vec{k})$ ) subject to the spherical normalization constraint $\sum_{m}\left[a_{q_{m}}\right]^{2}=N^{2}$. There are $(q L)^{d-1}$ 
amplitudes subject to a single normalization constraint and thus the ground state entropy scales as $(q L)^{d-1}$.

[47] In the notation of Eq. 20 for spin polarization independent spectra $v_{\alpha \beta}(\vec{k})=v(\vec{k}) \delta_{\alpha \beta}$, the partition function of the spherical (large $n$ ) model is

$$
Z=\prod_{\vec{k}}[\beta(v(\vec{k})+\mu)]^{-1 / 2},
$$

with $\mu$ determined from

$$
\beta=\int \frac{d^{d} k}{(2 \pi)^{d}}[v(\vec{k})+\mu]^{-1}
$$

with $\beta$ the inverse temperature. As is evident from Eqs. 2324 , for any of the $N$ ! permutations $P$ of the spectra $\{v(\vec{k})\} \rightarrow$ $\{v(P \vec{k})\}$, the partition function $Z$ is invariant. 